\title{
DUNLAP'S METHOD FOR THE MEAN VARIATION
}

\author{
BUFORD JOHNSON
}

Fram the Psychological Laboratory of the Bureau of Educational Experiments

In the Psychological Review for March, 1913, Knight Dunlap described a simple method developed by him, of obtaining the mean variation of a series of values, together with the necessary operations for carrying out the process on a calculating machine.

In the derivation of the formulae there is one obvious misprint in writing $(\Sigma P+P M)$ for $(\Sigma P-P M)$, but this is correctly printed when the same expression is used in the succeeding line.

The formulae derived are as follows:

$$
\begin{aligned}
& M V=(\Sigma P-P M) \div 1 / 2 N=(R M-\Sigma R) \div 1 / 2 N \text {, when } \\
& N=\text { total number of measures } \\
& M=\text { average or mean } \\
& M V=\text { mean variation } \\
& P \quad=\text { number of terms greater in value than the average } \\
& R=\text { number of terms less in value than the average } \\
& \Sigma P=\text { sum of the terms greater in value than the average } \\
& \Sigma R=\text { sum of the terms less in value than the average }
\end{aligned}
$$

These same formulae are reproduced, with a slightly different symbolization, in Whipple's ${ }^{1}$ Manual of Mental and Physical Tests, Part I: Simpler Processes, which was published in 1914. The following substitutes are used:

$$
\begin{array}{ll}
N_{+M} & \text { for } P \\
N_{-M} & \text { for } R \\
\Sigma_{+M} & \text { for } \Sigma P \\
\Sigma_{-M} & \text { for } \Sigma R \\
.5 & \text { for } 1 / 2
\end{array}
$$

1 Whipple's Manual of Mental and Physical Tests, Part I: Simpler Processes, p. 22. Dunlap developed the method itself and not merely the application of the calculating machine technique, as Whipple's footnote ( $p 21)$ might imply. We are not acquainted with any previous development of the method. 
Two alternative rules, based on the formulae, are given by Dunlap. In cases where all of the original terms employed are either positive or negative in sign, either of these two rules may be used. The second is a simpler one for operation on the machine. However, there are cases in which some of the terms averaged are positive and some are negative, the average being based on the algebraic sum of the terms. In these cases there may be difficulty in the application of rules (1) and (2), A third rule should be added which makes the procedure in such a case unmistakable. The procedure indicated in this third rule will avoid all danger of errors which may otherwise be serious.

The three rules which satisfy all the cases arising are as follows:

1. Add together the terms greater than the average; from the sum subtract the product of the number of terms $(P)$ so added, multiplied by the average $(M)$; and divide the remainder by half the total number of terms in the series $(1 / 2 N)$.

2. Add together the terms which are less than the average: subtract the sum from the product of the number of terms $(R)$ so added, multiplied by the average $(M)$; and divide the remainder by half the number of terms in the total series $(1 / 2 \mathrm{~N})$.

3 . In cases where the average is based on the algebraic sum of positive and negative terms, if this average is positive, compute the mean variation in accordance with Rule (1) from the positive terms which are numerically greater than the average. If the average is negative in sign, compute the mean variation in accordance with Rule (2) from the negative terms which are numerically greater than the average. 